Pacific Journal of Mathematics

年 


\title{
KO-EQUIVALENCES AND EXISTENCE OF NONSINGULAR BILINEAR MAPS
}

\author{
KeE YUEN LAM
}

\begin{abstract}
We show how to use homotopy theoretic methods to construct maps between various truncated projective spaces that induce isomorphisms in $K O$ cohomology theory. We then use these maps to establish the existence of new families of nonsingular bilinear maps.
\end{abstract}

1. Introduction. Bilinear maps $f: R^{a} \times R^{b} \rightarrow R^{c}$ with the nonsingular property that $f(x, y)=0$ implies $x=0$ or $y=0$ have been of interest for several reasons: (1) they generalize the multiplication map of the classical division algebras over $R$; (2) they provide estimates for the geometric dimension of vector bundles over real projective spaces [10], and are hence instrumental in the study of immersions of such spaces into $R^{n}$; (3) those maps with the additional property that $|f(x, y)|=|x| \cdot|y|$ can sometimes be used to produce essential harmonic maps between Euclidean spheres, in the sense of Eells and Sampson [5]. See [14] and [9, Theorem 4.2].

Furthermore, it has been realized for some time that there is an interesting relationship between nonsingular bilinear maps and stable homotopy theory [8], [9]. The purpose of this article is to further explore that relationship. Whereas [9] deals with known bilinear maps and their implications in homotopy theory, the present paper describes, in $\S 5$, how homotopy theory could in turn be used to establish the existence of new families of nonsingular bilinear maps. These new families are distinct from the classical examples of Hurwitz and Radon [7], and yet exhibit the same "Clifford periodicity" phenomenon which is characteristic of the Hurwitz-Radon family.

Since the Hurwitz-Radon family can be used to produce essential harmonic maps between Euclidean spheres, it would be interesting to ask whether there exist families of nonsingular bilinear maps, occurring in the same dimension ranges as the ones established in this paper, that will yield further examples of essential harmonic maps.

The main homotopy tools employed in the paper are certain maps between truncated projective spaces called $K O$-equivalences. Roughly, a $K O$-equivalence is a map inducing an isomorphism in $K O$-cohomology. The methods in $\S \S 3,4$ for constructing $K O$-equivalences follow closely the techniques of $[6, \S 4]$. Such constructions might have some independent interest, and could perhaps be read on their own right. 
2. Some homotopy lemmas. A finite connected $C W$ complex $X$ in which all cells apart from the base point occur in dimensions between $m$ and $n$ is written $X=X_{m}^{n}$. A typical example is the real truncated projective space $P_{m}^{n}=P^{n} / P^{m-1}$. We call $X_{m}^{n}$ stable if $n \leqq 2 m-1$, in which case $X_{m}^{n}$ is a suspension. We say that $X$ is 2-torsion, if $\widetilde{H}^{q}(X ; Z)$ is a 2-torsion group for all $q$. We shall also need the following standard notations:

$\mathscr{A}=$ the $\bmod 2$ Steenrod algebra;

$\mathscr{A}_{0}=$ the subalgebra of $\mathscr{A}$ generated by $S q^{1}$;

$\phi(n)=$ the number of integers in the interval $[1, n]$ congruent to $0,1,2$ or 4 modulo 8 .

Lemma 2.1. Suppose $X=X_{m}^{n}$ is a stable 2-torsion complex and that $\widetilde{H}^{*}\left(X ; Z_{2}\right)$ is free over $\mathscr{A}_{0}$. If $f: X \rightarrow X$ can be factored into a composite $f_{p} \cdots f_{2} f_{1}$, where $p>\phi(n-m)$ and each $f_{i}$ is a map $X \rightarrow X$ with induced homomorphism $f_{i}^{*}=0$ on $\tilde{H}^{*}\left(X ; Z_{2}\right)$, then $f$ is homotopic to zero.

Proof. Since $\tilde{H}\left(X ; Z_{2}\right)$ is $\mathscr{A}_{0}$-free, one can apply Adams' vanishing theorem [1, Thm. 2.1] to conclude that

$$
\operatorname{Ext}_{*}^{s, t}\left(\widetilde{H}^{*}\left(X ; Z_{2}\right), Z_{2}\right)=0 \text { for } s>\phi(t-s-m) \text {. }
$$

This means, in particular, that if

$$
X=E_{0} \longleftarrow E_{1} \longleftarrow E_{2} \longleftarrow \cdots \longleftarrow E_{s} \longleftarrow \cdots
$$

is a modified Postnikov tower over $X$ modelled on a minimal $\mathscr{A}$ free resolution of $\tilde{H}^{*}\left(X ; Z_{2}\right)$, as explained in [13, Chapter 18], then each $k$-invariant of dimension $\leqq n$ is located in $H^{*}\left(E_{s} ; Z_{2}\right)$ for some $s \leqq \phi(n-m)$. On the other hand, the hypothesis on $f_{i}^{*}$ allows one to prove inductively on $p$ that $f_{p} \cdots f_{2} f_{1}$ lifts to $E_{p}$. If $p>\phi(n-m)$, then all subsequent $k$-invariants will have dimension exceeding $n(=\operatorname{dim} X)$, and hence constitute no further obstruction to lifting $f_{p} \cdots f_{2} f_{1}$ arbitrarily high into the tower. Since the connectivity of $E_{s}$ eventually increases beyond any bound as $s \rightarrow \infty$, such lifting is enough to imply $f \simeq 0$.

CoROLlaRY 2.2. Let $\iota$ be the identity map of $X$ where $X$ is as in Lemma 2.1. Then the order of c, considered as an element in the track group $[X, X]$, is a divisor of $2^{\phi(n-m)+1}$.

CoROLlaRY 2.3. Let ke denote $k$ times the identity map $c$ in the track group $[X, X]$. If $X$ is as in Lemma 2.1, and $n-m \leqq 7$, then $16 \iota \simeq 0$. 
It goes without saying that by taking a closer look into the Postnikov tower of an individual $X$, it is sometimes possible to improve upon Corollary 2.2. A typical example is given by

Proposition 2.4. If $X$ is the truncated projective space $P_{m}^{m+5}$, where $m \geqq 9$ is congruent to 1 modulo 4 , then $8 \iota \cong 0$.

The proof is practically the same as Lemma 2.1, except that in the present case, $\operatorname{Ext}_{, . t}^{s, t}\left(H^{*}\left(P_{m}^{m+5} ; Z_{2}\right), Z_{2}\right)$ has somewhat better vanishing properties than enunciated by Adams' vanishing theorem. Alternatively, it is a routine exercise to write down a Postnikov tower for $P_{m}^{m+5}$ explicitly [13], and to check that $8 \iota=(2 \iota)(2 \iota)(2 \iota)$ lifts arbitrarily high into that tower.

Many of Toda's results in [15] on the "order of the identity class" of a suspension space are straightforward consequences of the above results.

\section{Construction of $\mathrm{KO}$-equivalences.}

Definition 3.1. A map $g: Y \rightarrow Z$ between two connected complexes is called a KO-equivalence if $g^{*}: K O(Z) \rightarrow K O(Y)$ is an isomorphism.

Notation 3.2. If $L$ is a skeleton of a truncated projective space $P_{m}^{n}$, the collapsing map from $P_{m}^{n}$ to $P_{m}^{n} / L$ will always be denoted by $c$. The inclusion map $L \rightarrow P_{m}^{n}$ will be denoted by $i, i^{\prime}, i^{\prime \prime}, j$, etc., or by an unmarked arrow.

THEOREM 3.3. Let $m$ be odd and $n$ be even. If $m+9 \leqq n \leqq$ $2 m-10$, then there exists a KO-equivalence $g: P_{m+8}^{n+8} \rightarrow P_{m}^{n}$ such that the composition

$$
P_{m}^{n+8} \stackrel{c}{\longrightarrow} P_{m+8}^{n+8} \stackrel{g}{\longrightarrow} P_{m}^{n} \stackrel{i}{\longrightarrow} P_{m}^{n+8}
$$

is equal to 16 .

Proof. Note that the hypotheses on $m$ and $n$ guarantee that all complexes appearing in $\left(^{*}\right)$ are 2 -torsion and stable, and that their mod 2 reduced cohomology rings are $\mathscr{A}_{0}$-free. If we make 16c: $P_{m}^{n+8} \rightarrow P_{m}^{n+8}$ cellular, and then collapse the $n$-skeleton of both its domain and range to a point, we get a map $P_{n+1}^{n+8} \rightarrow P_{n+1}^{n+8}$ which, being 16 times the identity, is trivial on account of Corollary 2.3. It follows that one can homotopically deform $16 \iota$ into a map $g_{0}: P_{m}^{n+8} \rightarrow P_{m}^{n}$. Since $m+7<n$ by hypothesis, we can further 
assume that throughout the course of the deformation, $P_{m}^{m+7}$ stays within $P_{m}^{n}$. It follows that the composite

$$
P_{m}^{m+7} \stackrel{i}{\longrightarrow} P_{m}^{n+8} \stackrel{g_{0}}{\longrightarrow} P_{m}^{n}
$$

is just a deformation of $P_{m}^{m+7} \stackrel{16 \ell}{\rightarrow} P_{m}^{m+7} \stackrel{i}{\rightarrow} P_{m}^{n}$, which is trivial by Corollary 2.3. Hence $g_{0}$ factors through the cofiber $P_{m+8}^{n+8}$ of $i$, and the resulting map is taken as $g$ in (*).

The $\widetilde{K O}$ groups of the complexes occurring in $\left(^{*}\right)$ are finite cyclic and 2-torsion. Since $i^{*}$ is onto and cokernel $e^{*}$ has order 16 , and $(i g c)^{*}$ is multiplication by $16, g^{*}$ must be an isomorphism in $K O$ theory. This concludes the proof.

REMARK 3.4. If $m$ and $n$ satisfy the hypothesis of the theorem, so would $m+8$ and $n+8$. Hence there is an infinite sequence of $K O$-equivalences

$$
\text { ... } \stackrel{g}{\longrightarrow} P_{m+8 k}^{n+8 k} \stackrel{g}{\longrightarrow} \ldots \stackrel{g}{\longrightarrow} P_{m+16}^{n+16} \stackrel{g}{\longrightarrow} P_{m+8}^{n+8} \stackrel{g}{\longrightarrow} P_{m}^{n} .
$$

REMARK 3.5. It is possible to use $2^{r} \iota,(r>4)$, rather than $16 \iota$, to construct $K O$-equivalences between truncated projective spaces. The approach is similar.

4. Further construction of $K O$-equivalences. The method of $\S 3$ can sometimes be adapted in order to construct a $K O$-equivalence $P_{m+8}^{n+8} \rightarrow P_{m}^{n}$, even if $m$ and $n$ do not satisfy the technical condition $m+9 \leqq n$ imposed in Theorem 3.3. However, a case by case presentation of such adaptations will be inelegant and unnecessary. For if $n-m=1,3,5$ or $7, P_{m+8}^{n+8}$ is homeomorphic to $\Sigma^{8} P_{m}^{n}$ [4]. Now Adams and Luz have a unified procedure for constructing $K O$-equivalences $\Sigma^{N} X \rightarrow X$ when $X$ is a suitable 2 -torsion finite complex and $N$ is a suitable power of 2 [2], [11]. Such a procedure applies nicely when $X=P_{m}^{n}$ with $n-m \leqq 7, m$ odd, $n$ even, provided that $m$ is not too small.

In this section we therefore confine our efforts to show how the ideas developed in $\S 3$ can be used to prove Theorem 4.1 below. This theorem has previously been obtained by Adams in $[2, \S 12]$, but we believe that it is of some interest to compare the two different proofs.

THEOREM 4.1. For any odd integer $m \geqq 9$ there is a KO-equivalence $g: P_{m+8}^{m+9} \rightarrow P_{m}^{m+1}$.

Proof. For $m \equiv 1(\bmod 8)$ and $m>9$, we construct $g$ by imita- 
ting the proof of Theorem 3.3. Thus we "compress" 16є: $P_{m}^{m+9} \rightarrow$ $P_{m}^{m+9}$ into a map $g_{0}: P_{m}^{m+9} \rightarrow P_{m}^{m+1}$, using a cellular homotopy. The following two lemmas imply that $g_{0}$ can be choosen such that its restriction to $P_{m}^{m+7}$ is trivial.

Lemma 4.2. For $m \equiv 1(\bmod 8)$ and $m>9$, the compression map $g_{0}$ can be chosen such that

$$
P_{m}^{m+7} \stackrel{i^{\prime}}{\longrightarrow} P_{m}^{m+9} \stackrel{g_{0}}{\longrightarrow} P_{m}^{m+1} \stackrel{i}{\longrightarrow} P_{m}^{m+7}
$$

is a trivial composite.

Lemma 4.3. Assume $m \equiv 1(\bmod 8)$ and $m>9$. Then a map $f: P_{m}^{m+7} \rightarrow P_{m}^{m+1}$ is trivial if and only if its composition with the inclusion $\operatorname{map} P_{m}^{m+1} \stackrel{i}{\rightarrow} P_{m}^{m+7}$ is trivial.

Postponing the proofs of these lemmas to the end of the section, we now form the cofiber map $g: P_{m+8}^{m+9} \rightarrow P_{m}^{m+1}$ induced by $g_{0}$. As in Theorem 3.3, $g$ is a $K O$-equivalence. In fact, it is not difficult to see that $g$ is at the same time a $K U$-equivalence and a $K S_{P}$-equivalence.

The case $m=9$ was excluded from the above, because $P_{m}^{m+9}$ fails to be stable when $m=9$. To handle this case, begin with a $K O$-equivalence $g: P_{25}^{26} \rightarrow P_{17}^{18}$, and consider its restriction $g^{\prime}: S^{25} \rightarrow P_{17}^{18}$. The Freudenthal theorem allows one to desuspend $g^{\prime}$ into a map $g^{\prime \prime}: S^{17} \rightarrow P_{9}^{10}$. But it is not hard to show that $\pi_{17}\left(P_{9}^{10}\right)$ is a direct sum of four copies of $Z_{2}$, so that $g^{\prime \prime}$ extends to a map $\hat{g}: P_{17}^{18} \rightarrow P_{9}^{10}$. The eight-fold suspension $\Sigma^{8} \hat{g}: P_{25}^{26} \rightarrow P_{17}^{18}$ agrees with $g$ on the bottom sphere $S^{25}$, and must therefore be a $K O$-equivalence because $g$ is such. By Bott periodicity, $\hat{g}$ is also a $K O$-equivalence.

We conclude by remarking that, in case the odd number $k$ is not congruent to $1(\bmod 8)$, a $K O$-equivalence $P_{k+8}^{k+9} \rightarrow P_{k}^{k+1}$ can still be constructed by suitably suspending one of the previously constructed $K O$-equivalences $g: P_{m+8}^{m+9} \rightarrow P_{m}^{m+1}$, with $m \equiv 1(\bmod 8)$.

REMARK 4.4. For a comparison between these $K O$-equivalences and those constructed by Adams in $[2, \S 12]$, the following observation is pertinent. While our construction involves a number of technical homotopy lemmas, it avoids Toda brackets and $e$-invariant considerations. Moreover, it falls into the general construction pattern of $\S 3$, and yields $K O$-equivalences $g: P_{m+8}^{m+9} \rightarrow P_{m}^{m+1}$ with the special property that

$$
P_{m}^{m+9} \stackrel{c}{\longrightarrow} P_{m+8}^{m+9} \stackrel{g}{\longrightarrow} P_{m}^{m+1} \stackrel{i}{\longrightarrow} P_{m}^{m+9}
$$


is exactly equal to 16 .

It is now time to prove Lemmas 4.2 and 4.3. The proof of 4.2 is based on the factorization $16 \iota=(2 \iota)(8 c)$. Consider the following homotopy commutative diagram

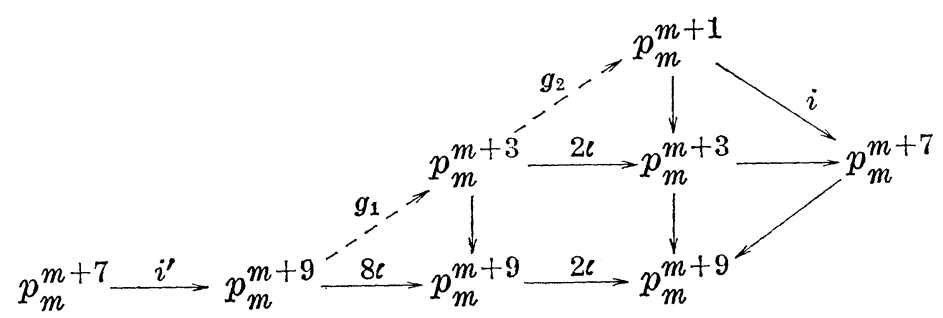

Here, $g_{1}$ exists because $8 \iota$ followed by the collapsing map $c: P_{m}^{m+9} \rightarrow$ $P_{m+4}^{m+9}$ is trivial (cf. Proposition 2.4). There are two obstructions to compressing $2 \iota$ into the map $g_{2}$. The first is $(2 \iota)^{*} x$, where $x$ generates $H^{m+2}\left(P_{m}^{m+3} ; Z_{2}\right)$. This obstruction obviously vanishes. The second obstruction is the functionalization of $S q^{2}$ on $x$ with respect to $2 \iota$. This vanishes because of indeterminacy.

We now pick $g_{0}=g_{2} g_{1}$ to be our compression of $16 c$, and proceed to show that $i g_{2} g_{1} i^{\prime}$ is trivial. To this end, notice that there is an alternative way to factorize $i g_{2} g_{1} i^{\prime}$, as given in the following commutative diagram:

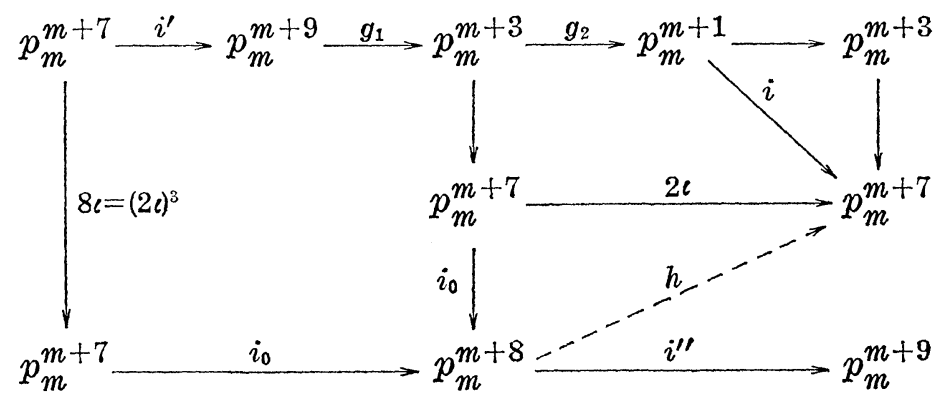

Since the two ways of going around the big square become homotopic when composed with $i^{\prime \prime}$, the big square itself is homotopy commutative for dimensional reasons. The dotted map $h$ exists because $P_{m}^{m+8}$ is obtained from $P_{m}^{m+7}$ via an attaching map of order 2 (cf. $\left[6\right.$, p. 58, bottom diagram]). Commutativity now gives $i g_{2} g_{1} i^{\prime}=$ $h i_{0}(8 \iota)=(2 \iota)(8 c)=16 \iota$, which is therefore trivial by Theorem 2.1.

The proof of Lemma 4.2 can be accomplished by showing that the corresponding dual statement is true. The Spanier-Whitehead duals of $P_{m}^{m+7}$ and $P_{m}^{m+1}$ can be taken as $P_{t}^{t+7}$ and $P_{t+6}^{t+7}$ respectively, 
where $t+m+8$ is a large power of $2($ see [4]), so that $t \equiv 7(\bmod$ 8 ) in the present context. From the hypothesized triviality of the composite

$$
P_{t}^{t+7} \stackrel{c}{\longrightarrow} P_{t+6}^{t+7} \stackrel{D f}{\longrightarrow} P_{t}^{t+7},
$$

we must now argue for the triviality of $D f$, the dual of $f$. It suffices to show that $D f$ lifts arbitrarily highly into the modified Postnikov tower

$$
P_{t}^{t+7} \longleftarrow E_{1} \longleftarrow E_{2} \longleftarrow E_{3} \longleftarrow \cdots
$$

over $P_{t}^{t+7}$. The following lists all the $k$-invariants of dimension $\leqq t+7$ arising in this tower, together with their defining relations, compare [12, p. 60]. (We use a subscript to indicate the dimension of a $k$-invariant.)

(0) primary $k$-invariant:

$$
\text { the generator } x_{t} \in H^{t}\left(P_{t}^{t+7} ; Z_{2}\right) \text {; }
$$

(1) $k$-invariants in $H^{*}\left(E_{1} ; Z_{2}\right)$ :

$$
\begin{aligned}
& k_{t+2}: S q^{2} S q^{1} x_{t}=0, \\
& k_{t+4}: S q^{4} S q^{1} x_{t}=0, \\
& k_{t+5}: S q^{4} S q^{2} x_{t}=0, \\
& k_{t+7}: S q^{8} x_{t}=0 ;
\end{aligned}
$$

(2) $k$-invariants in $H^{*}\left(E_{2} ; Z_{2}\right)$ :

$$
\begin{aligned}
& \kappa_{t+3}: S q^{2} k_{t+2}=0 \\
& \kappa_{t+4}: S q^{1} k_{t+4}+S q^{2} S q^{1} k_{t+2}=0, \\
& \kappa_{t+7}: S q^{4} k_{t+4}+S q^{6} k_{t+2}=0 ;
\end{aligned}
$$

(3) $k$-invariant in $H^{*}\left(E_{3} ; Z_{2}\right)$ :

$$
\theta_{t+4}: S q^{1} \kappa_{t+4}+S q^{2} \kappa_{t+3}=0 \text {. }
$$

With this list, it is no longer difficult to check that if any of these $k$-invariants constitute an obstruction to trivializing $D f$, then it is actually an obstruction to trivializing $(D f) c$. In this way Lemma 4.3 is obtained.

5. Existence of nonsingular bilinear maps. It has been remarked by $\mathrm{M}$. Mahowald that $K O$-equivalences can be used to estimate the geometric dimension of vector bundles over projective 
spaces, via a procedure which is also known to J. F. Adams. Specifically, consider the following diagram:

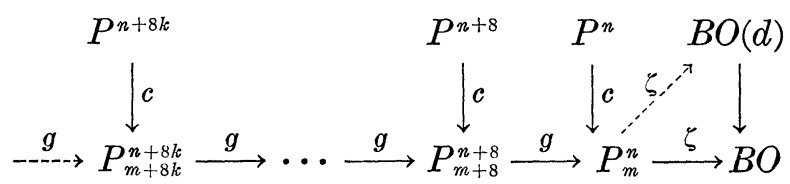

where $m, n$ are fixed integers, the $g$ 's are $K O$-equivalences, $\zeta$ represents the generator of $\widetilde{K O}\left(P_{m}^{n}\right)$, and $d$ is the geometric dimension of $\zeta$, that is, the smallest integer for which a lifting $\hat{\zeta}$ of $\zeta$ into $B O(d)$ exists. According to the computation of [3], $\widetilde{K O}\left(P_{m}^{n}\right)$ is cyclic of order $2^{\beta(m, n)}$, where $\phi(m, n)=\phi(n)-\phi(m-1)$. It follows that $\zeta g^{k} c=\zeta(g \cdots g) c$ can be interpreted as a stable vector bundle over $P^{n+8 k}$ represented by $\left(2^{\phi(n+8 k)} / 2^{\phi(m, n)}\right) \xi=2^{4 k+\phi(m-1)} \xi$, where $\xi$ denotes the Hopf line boundle. This stable bundle has geometric dimension $\leqq d$, due to the existence of $\hat{\zeta}$. As long as $2^{\phi(m-1)}>n$, the fact can be re-stated as:

$(\dagger) \quad 2^{4 k+\phi(m-1)} \xi$ over $P^{n+8 k}$ has at least $2^{4 k+\phi(m-1)}-d$ everywhere independent sections.

Notice that $d$ depends on $m$ and $n$ alone, and is independent of $k$.

To convert existence of sections into existence of nonsingular bilinear maps, we invoke the result of [8], which asserts that given $(\dagger)$, there is a (large) power of 2 , say $N_{0}$, such that for any positive multiple $q N_{0}$, a nonsingular bilinear map $R^{a} \times R^{c-d} \rightarrow R^{c}$ exists with $a=8 k+n+1$ and $c=2^{4 k+\phi(m-1)}+q N_{0}$ respectively. Letting $k$ run through $0,1,2, \cdots, l$ and picking $N$ to be the maximum of the $N_{0}$ 's so incurred, we obtain

THEOREM 5.1. Let $m, n$ be fixed integers satisfying $2^{\phi(m-1)}>n$, such that there exists a sequence of KO-equivalences

$$
\ldots \stackrel{g}{\longrightarrow} P_{m+8 k}^{n+8 k} \stackrel{g}{\longrightarrow} \ldots \stackrel{g}{\longrightarrow} P_{m+8}^{n+8} \stackrel{g}{\longrightarrow} P_{m}^{n} .
$$

Then for every $l>0$ there is a power of 2 , denoted by $N$, corresponding to which there exists a family of nonsingular bilinear maps

$$
\phi_{k}: R^{8 k+n+1} \times R^{2^{4 k+\phi(m-1)}+N-d} \longrightarrow R^{2^{4 k+\phi(m-1)}+N},
$$

with $k=0,1, \cdots, l$. Here $d$ depends only on $m$ and $n$, and is given by (\#) above. 
Let $a=8 k+n+1, \quad c=2^{4 k+\phi(m-1)}+N$ and $b=c-d$. The numerical features of the family $\phi_{k}: R^{a} \times R^{b} \rightarrow R^{\circ}(0 \leqq k \leqq l)$ becomes striking if we recall that the classical Hurwitz-Radon maps constitute a nonsingular family

$$
\Psi_{k}: R^{\alpha} \times R^{\beta} \longrightarrow R^{r}
$$

where $\alpha=8 k+2^{\varepsilon}, \beta=\gamma=2^{4 k+\varepsilon}$, with $\varepsilon=0,1,2$ or 3 and $k$ arbitrary. This family has the "Clifford periodicity" in that as $\beta$ and $\gamma$ are multiplied by $16, \alpha$ increases by 8 . If, by ignoring the constants $N$ and $d$, we introduce $a^{\prime}=8 k+n+1, b^{\prime}=2^{4 k+\phi(m-1)}, c^{\prime}=$ $2^{4 k+\phi(m-1)}$ and call $\left(a^{\prime}, b^{\prime}, c^{\prime}\right)$ the essential ranges of the family $\left\{\phi_{k}\right\}_{0 \leqq k \leq l}$, then we can summarize Theorem 5.1 above by saying that the essential ranges of $\left\{\phi_{k}\right\}$ exhibit the same Clifford periodicity as the dimension ranges $(\alpha, \beta, \gamma)$ of Hurwitz-Radon maps.

As an illustration let us specify diagram (\#) to the case $m=9$ and $n=10$, using the $K O$-equivalences $g$ constructed in $\S 4$. Using $\pi_{9}(B O(7))=Z_{2} \oplus Z_{2}$, it is not difficult to show that the generator $\zeta: P_{9}^{10} \rightarrow B O$ lifts (optimally) to $B O(7)$. Consequently we take $d=7$ in $(\#)$ to obtain

Special case 5.2. For every $l>0$ there is a power of 2 , denoted by $N$, corresponding to which there exists a family of nonsingular bilinear maps

$$
\phi_{h-1}: R^{8 h+3} \times R^{2^{4 h+N-7}} \longrightarrow R^{2^{4 h}+N}
$$

for $h=1,2, \cdots, l$.

Here for simplicity we have introduced $h$ to stand for $k+1$ in Theorem (5.1).

\section{REFERENCES}

1. J. F. Adams, A periodicity theorem in homological algebra, Proc. Camb. Phil. Soc., 62 (1966), 365-377.

2. - On the groups $J(X)-I V$, Topology, 5 (1966), 21-71.

3. - Vector fields on spheres, Ann. Math., 75 (1962), 603-632.

4. M. F. Atiyah, Thom complexes, Proc. Lond. Math. Soc., 11 (1961), 291-310.

5. J. Eells and J. H. Sampson, Harmonic maps of Riemannian manifolds, Amer. J. Math., 86 (1964), 109-160.

6. S. Feder, S. Gitler and K. Y. Lam, Composition properties of projective homotopy classes, Pacific J. Math., 68 (1977), 47-61.

7. A. Hurwitz, Über die Komposition der quadratischen Formen, Math. Ann., 88 (1923), 1-25.

8. K. Y. Lam, Nonsingular bilinear maps and stable homotopy classes of spheres, Proc. Camb. Phil. Soc., 82 (1977), 419-425.

9. _ـ Some interesting examples of nonsingular bilinear maps, Topology, 16 (1977), 185-188. 
10. K. Y. Lam, On bilinear and skew-linear maps that are nonsingular, Quart, J. Math. Oxford, (2) 19 (1968), 281-288.

11. C. G. C. Luz, Doctoral dissertation, Cambrige University, (1976).

12. M. Mahowald, The metastable homotopy of $S^{n}$, Memoir, Amer. Math. Soc. No. 72 (1967).

13. R. Mosher and M. T'angora, Cohomology Operations and Applications in Homotopy Theory, Harper and Row, New York, 1968.

14. R. T. Smith, Harmonic mappings of spheres, Amer. J. Math., 97 (1975), 364-385. 15. H. Toda, Order of the identity class of a suspension space, Ann. of Math., 78 (1963), 300-325.

Received November 27, 1977. Supported by an NRC grant of Canada. UNIVERSiTy of British Columbia

VANCOUver, B.C. Canada V6T 1W5 


\section{PACIFIC JOURNAL OF MATHEMATICS}

\section{EDITORS}

DoNALD BABBITT (Managing Editor)

University of California

Los Angeles, California 90024

HUGo Rossi

University of Utah

Salt Lake City, UT 84112

C. C. MOORE and ANDREW OGG

University of California

Berkeley, CA 94720
J. DUGUNDJI

Department of Mathematics University of Southern Californı Los Angeles, California 90007

R. Finn and J. Milgram Stanford University Stanford, California 94305

\section{ASSOCIATE EDITORS}

E. F. BECKENBACH

B. H. Neumann
F. WOLF

K. YOSHIDA

\section{SUPPORTING INSTITUTIONS}

UNIVERSITY OF BRITISH COLUMBIA CALIFORNIA INSTITUTE OF TECHNOLOGY UNIVERSITY OF CALIFORNIA MONTANA STATE UNIVERSITY UNIVERSITY OF NEVADA, RENO NEW MEXICO STATE UNIVERSITY OREGON STATE UNIVERSITY UNIVERSITY OF OREGON
UNIVERSITY OF SOUTHERN CALIFORNIA STANFORD UNIVERSITY UNIVERSITY OF HAWAII UNIVERSITY OF TOKYO UNIVERSITY OF UTAH WASHINGTON STATE UNIVERSITY UNIVERSITY OF WASHINGTON 


\section{Pacific Journal of Mathematics}

Vol. 82, No. $1 \quad$ January, 1979

Werner Bäni, Subspaces of positive definite inner product spaces of countable dimension ...................................... 1

Marilyn Breen, The dimension of the kernel of a planar set..............

Kenneth Alfred Byrd, Right self-injective rings whose essential right ideals

are two-sided

Patrick Cousot and Radhia Cousot, Constructive versions of Tarski's fixed

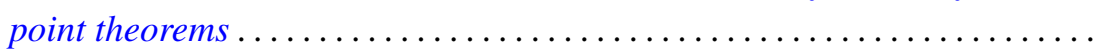

Ralph S. Freese, William A. Lampe and Walter Fuller Taylor, Congruence lattices of algebras of fixed similarity type. $I \ldots \ldots \ldots \ldots \ldots \ldots \ldots$

Cameron Gordon and Richard A. Litherland, On a theorem of Murasugi .....

Mauricio A. Gutiérrez, Concordance and homotopy. I. Fundamental

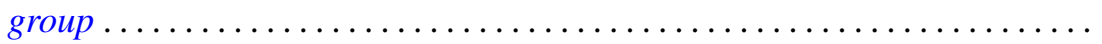

Richard I. Hartley, Metabelian representations of knot groups .............

Ted Hurley, Intersections of terms of polycentral series of free groups and free

Lie algebras ........................................

Roy Andrew Johnson, Some relationships between measures ............ 117

Oldřich Kowalski, On unitary automorphisms of solvable Lie algebras .......

Kee Yuen Lam, $K O$-equivalences and existence of nonsingular bilinear

maps...................................................

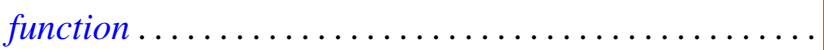

Robert A. Messer and Alden H. Wright, Embedding open 3-manifolds in compact 3-manifolds ............................

Gerald Ira Myerson, A combinatorial problem in finite fields. I . .

James Nelson, Jr. and Mohan S. Putcha, Word equations in a band of paths.

Baburao Govindrao Pachpatte and S. M. Singare, Discrete generalized Gronwall inequalities in three independent variables . .

William Lindall Paschke and Norberto Salinas, $C^{*}$-algebras associated with free products of groups ........................

Bruce Reznick, Banach spaces with polynomial norms ....

David Rusin, What is the probability that two elements of a finite group

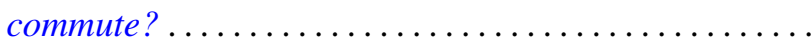

M. Shafii-Mousavi and Zbigniew Zielezny, On hypoelliptic differential operators of constant strength ...

Joseph Gail Stampfli, On selfadjoint derivation ranges .... . . .

Robert Charles Thompson, The case of equality in the matrix-valued triangle

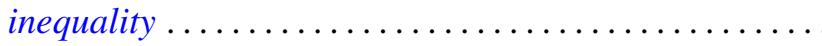

Marie Angela Vitulli, The obstruction of the formal moduli space in the negatively graded case. 